\title{
Data Hiding for Binary Documents Robust to Print-Scan, Photocopy and Geometric Distortions
}

\author{
Hae Yong $\mathrm{Kim}^{1}$ and Joceli Mayer ${ }^{2}$ \\ ${ }^{I}$ Escola Politécnica, Universidade de São Paulo,Brazil,hae@lps.usp.br. \\ ${ }^{2}$ LPDS/EEL, Universidade Federal de Santa Catarina, Brazil, mayer@eel.ufsc.br.
}

\begin{abstract}
This paper presents a data hiding technique for printed bicolor documents. It inserts tiny dots, hardly noticeable at normal reading distance, to embed the message. For message extraction, we employ autocorrelation and tiny registration dots to rectify geometric distortions. This technique is robust to distortions resulting from print-scan operations, good quality photocopies, affine transformations and scribblings/stains on the paper. The technique can be applied to documents with large white (or black) areas and they may present characters, drawings, schematics, diagrams, cartoons, but not halftones. The technique is intended to be neither a robust watermark (because any filtering can remove the dots) nor a covert communication (because the dots are perceptible at short distance). Nevertheless, when combined with a perceptual hashing and a cryptography protocol, it can be applied as semi-fragile authentication watermarking for hardcopy two-tone documents. In some situations, the utilization of the proposed system can substitute the use of notarial authenticated photocopies.
\end{abstract}

\section{Introduction}

This paper presents a data hiding technique (steganography) for embedding information into documents printed in high-resolution bicolor printers, e.g., conventional laser and inkjet printers. In the literature, there are several data hiding techniques designed for binary images. These techniques can be applied to copy control, annotation, and authentication. However, most of them are designed only for binary images in digital form and cannot be applied for printed documents. Data hiding for binary images can be divided into three basic classes:

1. Component-wise: Change the characteristics of some pixel groups (connected components, character, words, etc.) For example, the thickness of strokes, the position/area of characters/words [1], the brightness of the connected components [2], etc.

2. Pixel-wise: Change the values of individual pixels. Those pixels can be chosen randomly $[3,4]$ or according to some visual impact measure $[5,6]$.

3. Block-wise: Divide the cover image into blocks and modify some characteristic of each block to hide the data. Some papers suggest changing the parity or the quantization step of the number of black pixels in each block $[7,8]$. Others suggest flipping one specific pixel in the block with $m$ pixels to insert $\left\lfloor\log _{2}(m+1)\right\rfloor$ bits $[9,10]$.

Only a few of these techniques can be used to hide data in printed binary images, because print-scan operation introduces many distortions. For instance, geometrical misalignments are introduced by moving parts of the printer and by the user when placing the document at the scanner bed. Physical and chemical deformations of the paper are originated by high temperature and pressure of the toner fuser. Grayscale magnitude distortions are introduced by the optical and acquisition system of the scanner. Variable toner intensity distribution along the cylinder, paper texture and dirty introduces more distortions.

Most of data hiding techniques for printed binary images are component-wise and are designed only for a specific kind of image (for example, text documents) based on different approaches to modulate the message. Word or character shifting based techniques modulates the horizontal and/or vertical space between words or characters $[1,11,12]$. Character modulation schemes alter the character amplitude, texture or even the halftone $[2,13]$. 1-D and 2-D bar codes techniques propose to authenticate the text by modulating a visible image (barcode) introduced into the document [14, 15]. For many of these techniques, the message bit rate is severely reduced when the documents present few characters, large white (or black) areas, handwritings, drawings, equations and official stamps. Another drawback is that many techniques require a perfect segmentation (of connected components, characters or 
words), which is hard to achieve in practice due to distortions introduced by printing, scanning and nonmalicious geometric rotations.

$\mathrm{Wu}$ and Liu proposed a block-wise data hiding that can be applied for printed binary images in some specific scenarios [7]. However, this approach requires exceptionally high quality printing and scanning. It requires precise detection of the boundaries of the document to identify the size, rotation and skewing of the image. The reader is referred to $[2,7]$ for a discussion about watermarking for printed text and binary documents.

In the literature, there are many papers on continuous tone (grayscale and color) images watermarking techniques resilient to rotation, scale and translation (for example $[16,17,18]$ ) and continuous tone data hiding approaches resilient to print-scan (for example $[19,20])$. However, none of them can be directly applied to printed binary documents.

ry glance at ancient history shows clearly how different cultures, there arise at the same time t] nan life: Who am I? Where have I come from an : is there after this life? These are the questions $\mathrm{w}$ as also in the Veda and the Avesta; we find them and in the preaching of Tirthankara and Buddha; the tragedies of Euripides and Sophocles, as the (a)

ry glance at ancient history shows clearly how different cultures, there arise at the same time $\mathrm{t}$. nan life: Who am I? Where have I come from an is there after this life? These are the questions w as also in the Veda and the Avesta; we find them and in the preaching of Tirthankara and Buddha; the tragedies of Euripides and Sophocles, as the Fig. 1: Watermarked document at approximately normal resolution before (a) and after (b) print-scan operation.

This paper proposes a technique named DHDD (Data Hiding for Documents based on Dots). Current laser/inkjet printers can print tiny dots hardly noticeable at normal reading distance. Figure 1 depicts parts of a watermarked document before and after print-scan operation. We insert tiny dots, pseudo-randomly distributed over the entire document, to embed the message. Our current implementation is able to embed up to 1370 bits in an A4-sized document printed at 600 dpi. As the printing technology evolves, it is expected that future printers will be able to impress even smaller dots, resulting in more visually imperceptible watermarking with more data hiding capacity. We propose to use the entire binary document for embedding the watermark with a high robustness to print-scan, photocopy and geometric attacks.

\section{The DHDD technique}

We insert tiny dots, pseudo-randomly distributed over the entire document, to embed the message. In order to extract the message embedded by the proposed technique, the watermarked document is scanned and the tiny dots are detected. If the scanned image were not geometrically distorted, the data extraction would be an easy task. Unfortunately, geometric distortions (small rotation, translation, scale changing, etc.) are inherent to print-scan operations. Thus, we detect and compensate for affine transformations prior to the message extraction. For continuous-tone images, Kutter [16] suggested embedding the same watermark several times at horizontally and vertically shifted locations and to use auto-correlation to detect it. We employ a similar approach to address minor scaling and minor rotation: the document is segmented into four quadrants and the same watermark is embedded into each quadrant. Unfortunately, the autocorrelation approach is unable to withstand translation, cropping or a major rotation (90, 180 or 270 degrees). We propose to detect and compensate for these distortions by inserting some extra registration dots.

The resulting technique is robust to print-scanning, good quality photocopying, geometric attacks, cropping and scribbling/stains on the paper. It can be applied to documents containing characters, drawings, schematics, cartoons, logos, equations and also to documents with large white (or black) areas, as we can embed black dots over white background or viceversa. However, documents containing halftone elements cannot be watermarked by DHDD, because the resulting halftone patterns cannot be distinguished from the inserted tiny dots.

DHDD, working together with a perceptual hashing and a public key cryptography, can achieve a complete bicolor hardcopy document authentication system, that can substitute the use of notarial authenticated photocopies. Unfortunately, still there is no reliable perceptual hashing available for document authentication.

\section{Data insertion}

Figure 2 depicts the data insertion process. Given the original document, the sequence of bits to be hidden, and a seed of the pseudo-random number genera- 
tor, the data insertion process consists on obtaining the watermarked document (a binary image) to be printed.

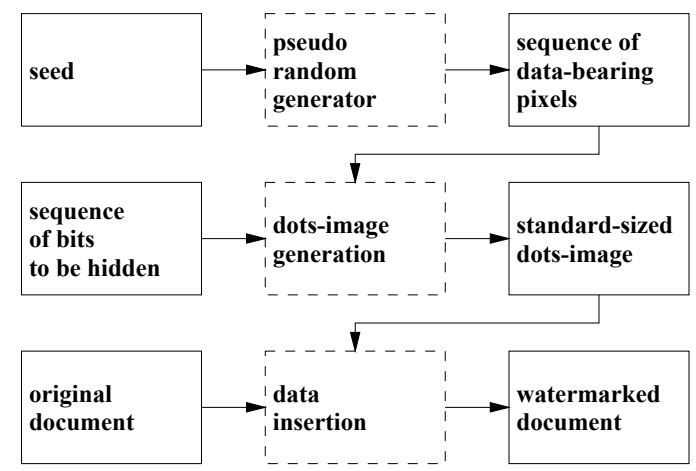

Fig. 2: Data insertion.

The "dots-image" with $t_{w} \times t_{w}$ pixels (Figure 3a) is an important internal data structure in both data insertion and data extraction phases. Its size $\left(t_{w} \times t_{w}=1024 \times 1024\right.$ in our implementation $)$ is a parameter known by both the data insertion and extraction algorithms. The dots-image indicates in black the spatial positions where the tiny dots must be inserted in the watermarked document (Figures $3 \mathrm{~b}$ and 1). In our implementation, the dots-image is divided in 4 quadrants. Each quadrant has $t_{2} \times t_{2}$ pixels, where $t_{2}=t_{w} / 2$. Each quadrant receives the same pattern of dots (Figure 4). Thus, the data extraction algorithm can detect and compensate for rotation and scaling distortions.

The pseudo-random generator chooses a sequence of $n_{q}$ data-bearing pixels for each quadrant, where $n_{q}=6144$ in our implementation. A given distance separates these pixels (at least 5 pixels, in our implementation), in order to assure that no pair of dots gets merged during the print-copy-scan operations. Each chosen pixel can be set (black in dots-image, indicating the insertion of a tiny dot) or reset (white in dotsimage, indicating the absence of the dot). In our implementation, each hidden bit is represented by 4 databearing pixels in each quadrant (or 16 data-bearing pixels in the whole dots-image, see Figure 4). Using more data-bearing pixels per bit, fewer bits can be embedded but the robustness is increased, and vice versa. In our implementation, bit 0 is represented by the sequence of data-bearing pixels BWBW ( $\mathrm{B}=$ black and $\mathrm{W}=$ white) and bit 1 is represented by the sequence WBWB. Figure 4 depicts a possible configuration of the 16 data-bearing pixels that hides one bit. If pixels 0 and 2 are black and 1 and 3 are white, the hidden bit is 0 . If pixels 0 and 2 are white and 1 and 3 are black, the hidden bit is 1 . For simplicity, we are using a squareshaped dots-image. Choosing a dots-image with the

same aspect ratio of the document to be watermarked (A4, letter or other) would increase the number of bits that can be hidden. Only the black data-bearing pixels will be detected through the corresponding tiny dot. The white data-bearing pixels are represented by the absence of the tiny dot in the data-bearing location.

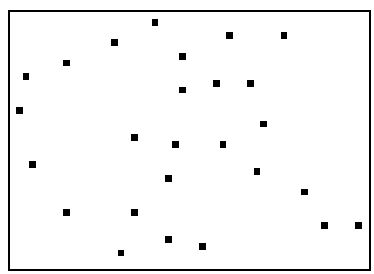

(a) Part of the standard sized dots-image.

\section{print-scan)}

Fig. 3: Some intermediary images of the data insertion.

As there are $n_{q}=6144$ data-bearing pixels per quadrant in our implementation, a total of $n_{q} / 4=1536$ bits can be embedded into the document. However, 16 of these bits are used to indicate the length of the hidden sequence and $n_{s}$ bits (150 in our case) are used as registration/synchronization dots to detect and compensate for the translation and major rotation. Therefore, the net length of the hidden sequence of bits is $n_{l}=\left(n_{q} / 4\right)-16-n_{s}$, or 1370 bits in our implementation.

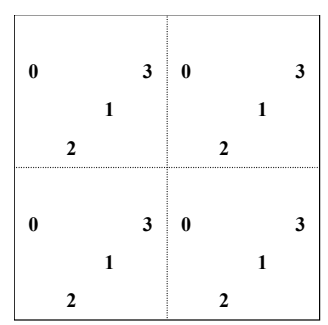

Fig. 4: A dots-image with 16 data-bearing pixels that represent one bit. The image is divided in 4 quadrants and each quadrant receives the same pattern of databearing pixels.

The dots-image (Figure $3 a)$ is the reference to embed the tiny dots in the document (Figure 3b). The watermarked document usually has a much larger resolution than the dots-image; for example, an A4 document at 600 dpi has more or less $6600 \times 4400$ pixels in the printable area. Thus, the dots-image must be conveniently scaled, before generating the watermarked document.

The tiny dots in the watermarked document can be either black over white background or white inside a black character. We have observed a practical limit for 
the size of the small isolated dots. The printers we tested (Oki B4350 and Brother HL-1440) could not print black dots smaller than $2 \times 2$ pixels or white dots smaller than $3 \times 3$ pixels, at $600 \mathrm{dpi}$. Thus, each dot in the watermarked document is defined with one of these limiting sizes.

We avoid placing dots near the borders of characters, because the borders of characters may become blurred by the print-scan operations and thus a dot situated at the edge of a character may not be properly detected. So, the data insertion algorithm searches, for each black pixel in the dots-image, the nearest safe location in the document image. The watermark extraction algorithm uses a low-pass filter to "enlarge" the sizes of the tiny dots, in order to deal with small position shiftings. In our implementation, a safe pixel in a black document region (where a white dot will be inserted) is situated at least 5 pixels away from the character borders, and a safe pixel in a white region is situated at least 6 pixels away from the border. Sometimes, when no safe location can be found, the dot can be discarded due to the redundancy of the technique provided by the repetition code and due to the watermark replication on the four quadrants. Figure 1 depicts the appearance of a watermarked document before and after the print-scan operation.

\section{Data extraction}

Figure 5 depicts the data extraction process. Given the scanned watermarked document (Figures $1 \mathrm{~b}$ and $6 a)$ and the seed of the pseudo-random generator (the same as in the insertion), the data extraction process consists on recovering the hidden bits.

The data extraction begins by detecting the tiny isolated dots, yielding the "high-resolution dotsimage" (Figure 6b). Many different algorithms can be used to detect the tiny dots. We use a very simple strategy: a pixel $p$ is classified as an isolated tiny dot when its grayscale value is sufficiently brighter or darker than all the neighbor pixels situated at distance 4 from $p$ (using the chessboard distance or 8connectivity), provided that the grayscale of pixel $p$ is within an acceptable range. These detected isolated dots may form small clusters. For each connected component, we classify only its central pixel as a truly isolated dot. The obtained image is resized (using a special resizing algorithm that does not lose any black pixel) to yield a standard-sized (but possibly still geometrically distorted) dots-image (Figure 6c).

The aim of the following operations is to detect and compensate for the geometrical distortions. The autocorrelation of the standard-sized dots-image will be used to detect the parameters of rotation, scaling and shearing. However, as we know only the positions of black data-bearing pixels (white ones cannot be localized), this image cannot be directly auto-correlated, requiring some preprocessing. First, the margins of the standard-sized dots-image must be detected, in order to avoid their interference in the auto-correlation. These margins are characterized by the absence of the tiny dots. We detect the margins using a morphological "closing" operation with $35 \times 35$ structuring element and another filter that eliminates small connectedcomponents. The result of this operation is an image completely white in the margins and completely black in the printed area, which allows us to classify any pixel as belonging to either "margin area" or "printed area." Then, the standard-sized dots-image is low-pass filtered, and the average gray level of the pixels in the printable area is computed. The pixels in the printed area have their values subtracted by the average value, and the pixels in the margin area are set to zero. Figure $6 \mathrm{~d}$ depicts this image, where the dark, medium and light shades of gray represent respectively negative, zero and positive values.

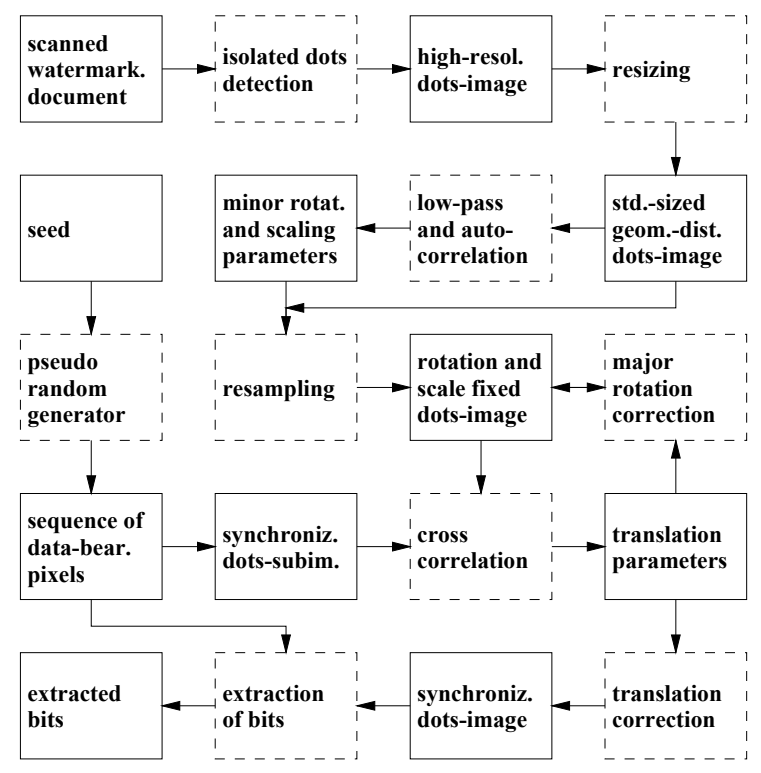

Fig 5: Data extraction

This image is auto-correlated, yielding an image with 9 peaks. Figure 6e depicts the nine peaks of a non-rotated stego image, and Figure $6 \mathrm{f}$ depicts the nine peaks of a rotated stego image. The auto-correlation can be computed efficiently using FFT (Fast Fourier Transform) and the correlation theorem. The autocorrelation of the "high-resolution dots-image" would be quite time-consuming, and this is one of the reasons 
why we resized it to a smaller standard size $(1024 \times 1024$ pixels $)$. The nine peaks are much stronger and clearer than in continuous-tone watermarking [16], because in our process there is no interference of the host image. Thus, we obtained a high robustness even using only the four corner peaks to detect rotation, scaling and shearing parameters. We use the locations of these four corner peaks, and the locations where these four peaks should be without the geometric distortions, to perform a bilinear resampling, yielding the "rotation and scale fixed dots-image".

The obtained image may be rotated by 90,180 or 270 degrees. To detect the translation and major rotation parameters, we first generate the "synchronization dots-subimage" with $t_{2} \times t_{2}$ pixels $(512 \times 512$ in our implementation). All pixels of this image are zeroes except in the $4 \times n_{s}(4 \times 150$, in our case) registration databearing pixels where the pixel values are -1 (if the data-bearing pixel is black) or +1 (white). This image is low-pass filtered (Figure 6g). This image is crosscorrelated with the "rotation and scale fixed dotsimage." The resulting image must contain four peaks that form a square with sides of approximate $t_{2}$ pixels (512 in our case, Figure 6h), unless the image has undergone a major rotation. In this case, the four peaks will not be detected and the "rotation and scale fixed dots-image" is rotated by $90^{\circ}$ (then by $180^{\circ}$ and $270^{\circ}$ ) and cross-correlated again until detecting the four peaks. These trial rotations end up by identifying the major rotation parameter. After identifying the major rotation parameter, we use the positions of the four peaks and where they should be without the translation, to detect and compensate for the translation, yielding the "synchronized dots-image." This image is low-pass filtered, because the dots may not be positioned exactly at the data-bearing locations. To extract a bit $b$, the sequence of the 16 data-bearing pixel values corresponding to $b$ (extracted from the filtered synchronized dots-image by accessing the expected positions of the data-bearing pixels) is correlated with the sequence $(+1,-1,+1,-1,+1,-1,+1,-1,+1,-1,+1$, $-1,+1,-1,+1,-1)$. The signal of the correlation coefficient $r$ indicates if the hidden bit is 0 or 1 . If $|r|$ is too small, the extracted bit may be erroneous. We warn the user that the extracted bit can be incorrect if $|r|<0.08$.

\section{Experimental results}

\subsection{Preliminary tests}

We adjusted the parameters of our implementation to use the Oki Data B4350 as the printing device and the Lexmark X83 as the scanning device, both working

at $600 \mathrm{dpi}$. We have tested also the Brother printer HL1440 and the HP 3400C. We have used the Xerox WorkCenter Pro 420 as the photocopy machine.

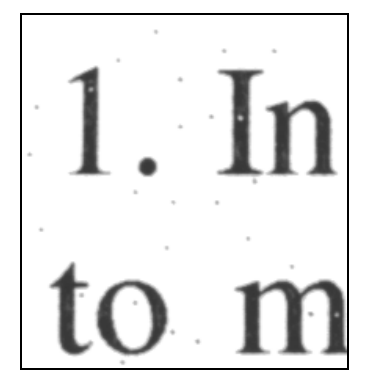

(a) Part of the scanned image.

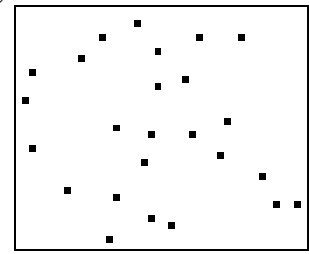

(c) Part of the standard-sized geometrically distorted dotsimage.

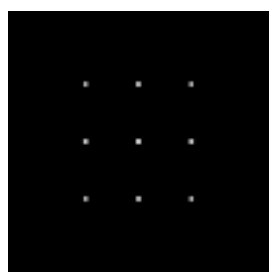

(e) The nine peaks of the auto-correlation image (dilated to improve visibility).

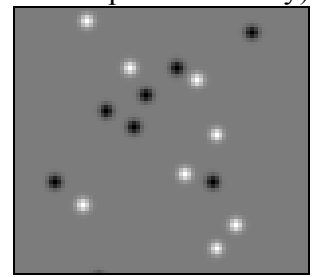

(g) Part of the low-pass filtered synchronization dotssubimage. Dark and light grays represent negative and positive values.

Fig. 6: Intermediary images of the data extraction.

We tested the proposed technique in two documents. An MS-Word document written in "Times New Roman 12 pts" (document A) and a page of an electronic version (PDF) of the IEEE T. PAMI containing a non-halftone figure and some equations (document B) were converted in two 600 dpi binary images. 1344 (d) Low-passed dots-image with the margins. Dark and light grays represent negative and positive values.

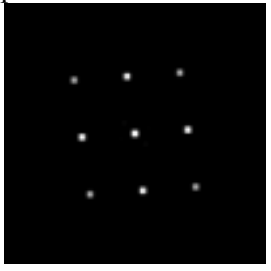

(f) The nine peaks of the auto-correlation obtained from a rotated stego image.

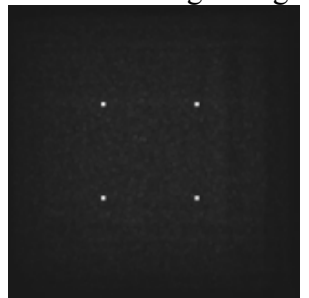

(h) The four peaks of the cross-correlation (dilated to improve visibility). 
bits were embedded in each binary image. Some black data-bearing pixels could not be translated into tiny dots because no safe location for them could be found in the cover images. Specifically, $1.9 \%$ of the black data-bearing pixels in document $\mathrm{A}$ and $5 \%$ in document B could not be transformed in tiny dots.

First, we tested extracting the hidden data from the watermarked binary documents $\mathrm{A}$ and $\mathrm{B}$ (without print-scanning them). The results were successful. Then, we printed the both watermarked images using the Oki printer and scanned them with the Lexmark scanner at $600 \mathrm{dpi}$, and all bits were correctly extracted. Then, we scanned the two documents with slight rotations, and the data were correctly extracted. We scribbled some handwritings on the documents, scanned them, resulting in perfect detection.

To test the resistance to different scalings, the two printed documents were scanned at 500 and 700 dpi, and the extractions were successful. To test the nonuniform scaling, the two images at 700 dpi were downsized to $600 \times 500 \mathrm{dpi}$ and the extractions were successful. To evaluate the robustness to rotations, we rotated the two documents 30 and 135 degrees using bilinear resampling. All the four extractions were successful. We applied cropping at different borders of the documents. We cropped $10 \%, 20 \%, 30 \%$ and $40 \%$ of the documents and all extractions were successful. The extractions failed only when cropping over $40 \%$ of the print-scanned documents. However, Stirmark tests indicate that DHDD is not so robust against cropping, using photocopies of the watermarked documents (next subsection). We also tested painting parts of the documents with black squares. Painting up to $30 \%$ of the document areas resulted in successful extractions. Painting over $40 \%$ of the document resulted in detection errors. We photocopied the printed documents using Xerox photocopier, scanned them, and the bits were correctly extracted. Similar results were obtained using Brother printer and HP scanner.

Typical processing time to insert data to an A4sized document is 12 seconds and the data extraction takes 110 seconds, using a $3 \mathrm{GHz}$ Pentium-4 computer.

\subsection{Stirmark tests}

Stirmark is a software used to test the robustness of watermarking techniques $[21,22]$. We carried on more exhaustive tests using documents distorted by Stirmark 4.0, release 129. We distorted 6 watermarked documents using Stirmark: the original binary documents A1 and B1 (before the print-scan operations); the grayscale images A2 and B2 obtained by printscanning documents $\mathrm{A} 1$ and $\mathrm{B} 1$; the grayscale images A3 and B3 obtained by print-photocopy-scanning documents A1 and B1. Each one of these 6 images was distorted in 90 different ways, using the default settings that come with the software. From the original settings, we discarded some tests that do not apply to our case (PSNR, Embed-Time and Self-Similarity). We inserted more parameters in Cropping $(80 \%$ to 95\%). We modified the parameters Convolution-Filter, because the original ones change the mean grayscale. The results are depicted in Table 1 . We can conclude that:

- DHDD is very robust against affine transforms, rotations, rotations followed by small cropping, and rotations followed by small scaling. Each image was distorted in 38 different ways and the extractions were always successful.

- Surprisingly, DHDD is robust against random removal of rows and columns of the image. One in each 10 to 100 rows and columns were removed and all extractions were always successful.

- DHDD is robust against JPEG compression, using 35 or higher quality parameter.

- DHDD is robust against rescaling, from $75 \%$ to $110 \%$.

- DHDD is robust against cropping using the original or print-scanned images, keeping $75 \%$ or more of the original documents' areas. DHDD is not robust against cropping of photocopied images.

- In some cases, DHDD can resist convolution with Gaussian and sharpening kernels.

- As expected, DHDD is not robust against median filtering and noise.

\subsection{Inkjet tests}

Finally, we tested our technique using Epson Stylus CX4900 all-in-one (scanner, photocopier and inkjet printer). We watermarked 8 different A4 documents, printed them in CX4900 using glossy papers, scanned them, extracted the hidden bits, and all extractions were correct. Then, we photocopied the 8 watermarked documents using CX4900 and glossy papers, scanned them, and successfully extracted the hidden bits.

We took one of the documents, and copied it successively, until obtaining incorrect bit extraction. After 3 successive copies 4 bits were extracted incorrectly. The tiny dots become larger and larger with successive photocopies. 
Tab. 1: Data extraction from images distorted by Stirmark 4.0. The symbol ''PE- $n$ ' means partial error with $n$ wrong bits (out of 1344 total bits); 'PE-*' means partial error with 10 or more wrong bits; 'TE' means that the bits extraction could not be performed; ' $k \times$ transform' means that the "transform" were applied using $k$ different parameters.

\begin{tabular}{|c|c|c|c|c|c|c|}
\hline & A1 & A2 & A3 & B1 & B2 & B3 \\
\hline $8 \times$ affine & - & - & - & - & - & - \\
\hline $10 \times$ rotcrop & - & - & - & - & - & - \\
\hline $10 \times$ rotscale & - & - & - & - & - & - \\
\hline $10 \times$ rotation & - & - & - & - & - & - \\
\hline $10 \times$ remove lines & - & - & - & - & - & - \\
\hline jpeg_15 & $\mathrm{TE}$ & PE-3 & PE-* & $\mathrm{TE}$ & - & PE-* \\
\hline jpeg 20 & TE & - & PE-2 & TE & - & PE-3 \\
\hline jpeg_25 & TE & - & PE-1 & PE-4 & - & PE-3 \\
\hline jpeg_30 & $\mathrm{TE}$ & - & - & PE-6 & - & - \\
\hline $8 \times$ jpeg ( 35 to 100$)$ & - & 一 & - & - & - & - \\
\hline resc_50 & - & $\mathrm{TE}$ & PE-6 & - & $\mathrm{TE}$ & $\mathrm{TE}$ \\
\hline resc_75 & - & - & - & - & - & - \\
\hline resc_90 & - & - & - & - & - & - \\
\hline resc_110 & - & - & - & - & - & - \\
\hline cropping_25 & $\mathrm{TE}$ & $\mathrm{TE}$ & $\mathrm{TE}$ & $\mathrm{TE}$ & $\mathrm{TE}$ & $\mathrm{TE}$ \\
\hline cropping_50 & $\mathrm{TE}$ & $\mathrm{TE}$ & TE & $\mathrm{TE}$ & $\mathrm{TE}$ & $\mathrm{TE}$ \\
\hline cropping_75 & 一 & - & PE-2 & - & - & PE-* \\
\hline cropping_80 & - & - & PE-1 & - & - & PE-* \\
\hline cropping_85 & - & - & - & - & - & PE-6 \\
\hline cropping_90 & - & - & - & - & - & PE-5 \\
\hline cropping_95 & - & - & - & - & - & - \\
\hline conv ( $3 \times 3$ Gaussian) & - & - & PE-5 & - & - & PE-5 \\
\hline conv $(3 \times 3$ sharpening $)$ & - & - & - & - & - & - \\
\hline median_3 & TE & PE-6 & PE-2 & $\mathrm{TE}$ & - & PE-2 \\
\hline median_5 & $\mathrm{TE}$ & $\mathrm{TE}$ & $\mathrm{TE}$ & $\mathrm{TE}$ & $\mathrm{TE}$ & $\mathrm{TE}$ \\
\hline median_7 & $\mathrm{TE}$ & $\mathrm{TE}$ & $\mathrm{TE}$ & $\mathrm{TE}$ & $\mathrm{TE}$ & $\mathrm{TE}$ \\
\hline median_9 & $\mathrm{TE}$ & $\mathrm{TE}$ & $\mathrm{TE}$ & $\mathrm{TE}$ & $\mathrm{TE}$ & $\mathrm{TE}$ \\
\hline noise_4 & - & $\mathrm{TE}$ & $\mathrm{TE}$ & - & $\mathrm{TE}$ & $\mathrm{TE}$ \\
\hline $4 \times$ noise $(8$ to 20$)$ & $\mathrm{TE}$ & TE & TE & TE & TE & $\mathrm{TE}$ \\
\hline rnddist_0.95 & PE-2 & PE-* & $\mathrm{TE}$ & PE-* & PE-* & PE-* \\
\hline rnddist_1.05 & $\mathrm{TE}$ & PE-* & $\mathrm{TE}$ & PE-* & PE-* & PE-* \\
\hline rnddist_1.1 & PE-3 & PE-* & $\mathrm{TE}$ & PE-* & PE-* & PE-* \\
\hline rnddist_1 & PE-7 & PE-* & TE & PE-* & PE-* & PE-* \\
\hline latestrnddist_0.95 & - & - & - & PE-3 & - & - \\
\hline latestrnddist_1.05 & - & PE-2 & - & PE-4 & - & - \\
\hline latestrnddist_1.1 & - & PE-1 & - & PE-6 & - & - \\
\hline latestrnddist_1 & - & - & - & PE-4 & - & - \\
\hline
\end{tabular}

\section{Authenticated photocopy}

Semi-fragile authentication watermarking is used to verify the originator of the image and to certify that its visual content has not been changed maliciously or accidentally. However, semi-fragile watermarking must not detect harmless alterations of the image (such as those generated by lossy compression, brightness/contrast adjusting, etc.) as image adulterations.

A semi-fragile watermarking typically uses a perceptual image hashing (also called robust visual hashing or media hashing $[23,24])$. The perceptual hashing
$h(A)$ is a value that identifies the image $A$. Moreover, given two images $A$ and $B$, the distance $D$ between the hashing $D[h(A), h(B)]$ must be somehow proportional to the perceptual visual difference of the images $A$ and $B$. Unfortunately, perceptual hashing for document authentication is still an ongoing research subject and there is no widely accepted standard. However, in the special scenario where the documents to be authenticated contain only characters, an OCR followed by a one-way cryptographic hashing can be used as a Boolean perceptual hashing function. In this case, the function $D$ assumes one of the two possible values: 0 if $A$ and $B$ are visually equivalent and 1 if they are not equivalent.

Let us suppose that Alice wants to authenticate a document $A$. She computes the hashing $h(A)$ and encodes it using her private-key, yielding the digital signature $D S(A)$. She embeds $D S(A)$ into the document using DHDD. She prints the watermarked document and sends it to Bob.

Bob receives the printed document and scans it, resulting in scanned image $B$. He extracts the hidden digital signature $D S(A)$ from the scanned image $B$ using DHDD and decodes it using the Alice's publickey, obtaining the extracted hashing value $h(A)$. If the data extraction is unsuccessful, the document is deemed not authentic, because it has undergone distortions strong enough to hinder extracting the hidden bits. Bob also filters out the data-bearing tiny dots from the scanned document $B$, obtaining the filtered document $\hat{B}$. Bob computes the perceptual hashing $h(\hat{B})$ of the filtered document. If $D[h(A), h(\hat{B})]=0$, the printed document is considered authentic.

Bob makes a good quality photocopy of the printed document. Bob send to Carol the photocopy of the document. Carol can verify the authenticity of the photocopy (that Alice generated the document and it has not been modified) by the same means.

\section{Conclusions}

We have presented a data hiding technique for printed binary documents. Tiny barely visible dots are used both to recover synchronism and to carry the information. Experiments illustrate the method's robustness to printing-scanning operations, good quality photocopying, affine transformations, cropping and scribbling/stains on the paper. We have described its application to semi-fragile authentication of printed documents. 


\section{References}

[1] N.F. Maxemchuk, and S. Low, "Marking Text Documents," IEEE Int. Conf. Image Processing, vol. 3, pp. 1317, 1997.

[2] P.V.K. Borges, J. Mayer, "Text Luminance Modulation for Hardcopy Watermarking”, Signal Processing Magazine, Elsevier, 2007.

[3] M.S. Fu, and O.C. Au, "Data Hiding by Smart Pair Toggling for Halftone Images," IEEE Int. Conf. Acoustics Speech and Signal Processing, vol. 4, pp. 2318-2321, 2000.

[4] H.Y. Kim, and A. Afif, "Secure Authentication Watermarking for Halftone and Binary Images," Int. J. Imaging Systems and Technology, vol. 14, no. 4, pp. 147-152, 2004.

[5] H.Y. Kim, "A New Public-Key Authentication Watermarking for Binary Document Images Resistant to Parity Attacks," IEEE Int. Conf. on Image Processing, vol. 2, pp. 1074-1077, 2005.

[6] Q. Mei, E.K. Wong, and N. Memon, "Data Hiding in Binary Text Documents," Proceedings of SPIE, vol. 4314, pp. 369-375, August 2001.

[7] M. Wu and B. Liu, "Data Hiding in Binary Image for Authentication and Annotation", IEEE Trans. on Multimedia, vol. 6, no. 4, pp. 528-538, Aug. 2004.

[8] H.Y. Kim and R.L. de Queiroz, "Alteration-Locating Authentication Water-marking for Binary Images," Int. Workshop on Digital Watermarking, (Seoul), Lecture Notes in Computer Science 3304, pp. 125-136, 2004.

[9] Y.C. Tseng, Y.Y. Chen, and H.K. Pan, "A Secure Data Hiding Scheme for Binary Images," IEEE Trans. on Communications, vol. 50, no. 8, pp.1227-1231, Aug. 2002.

[10] C.-C. Chang, C.-S. Tseng, and C.-C. Lin, "Hiding Data in Binary Images," Lecture Notes in Computer Science 3439, pp. 338-349, 2005.

[11] J.T. Brassil, S. Low, N.F. Maxemchuk, "Copyright Protection for the Electronic Distribution of Text Documents," Proc. of IEEE, vol. 87, no. 7, pp. 1181-1196, July 1999.

[12] D. Huang and H. Yan, "Interword Distance Changes Represented by Sine Waves for Watermarking Text Images", IEEE Trans. on Circuits and Systems for Video Technology, vol. 11, no. 12, pp 1237-1245, Dec. 2001.

[13] R. Víllan, S. Voloshynovskiy, O. Koval, J. Vila, E. Topak, F. Deguillaume, Y. Rytsar and T. Pun, "Text datahiding for digital and printed documents: theoretical and practical considerations", in Proc. of SPIE, Elect. Imaging, USA, 2006.
[14] R. Víllan, S. Voloshynovskiy, O. Koyal and T. Pun, "Multilevel 2D Bar Codes: Towards High Capacity Storage Modules for Multimedia Security and Management", in Proc. of SPIE, Elect. Imaging, USA, 2005.

[15] N.D.Quintela and F. Pérez-González, "Visible Encryption: Using Paper as a Secure Channel", in Proc. of SPIE, Elect. Imaging, USA, 2003.

[16] M. Kutter, "Watermarking Resisting to Translation, Rotation, and Scaling," SPIE Conf. Multimedia Syst. and App., vol. 3528, pp. 423-431, Nov. 1998.

[17] C.Y. Lin, M. Wu, J.A. Bloom, I.J. Cox, M.L. Miller and Y.M. Lui, "Rotation, Scale, and Translation Resilient Watermarking for Images," IEEE T. Image Processing, vol. 10 , no. 5, pp. 767-782, May 2001.

[18] S. Pereira, J.J.K.O. Ruanaidh, F. Deguillaume, G. Csurka, and T. Pun, "Template Based Recovery of FourierBased Watermarks Using Log-Polar and Log-Log Maps," IEEE Int. Conf. Multimedia Comp. Systems, vol. 1, pp. 870-874, Jun. 1999.

[19] K. Solanki, U. Madhow, B. S. Manjunath, S. Chandrasekaran, and I. El-Khalil, "Print and Scan Resilient Data Hiding in Images," IEEE T. Information Forensics and Security, vol. 1, no. 4, pp. 464-478, Dec. 2006.

[20] C.-Y. Lin, "Public Watermarking Surviving General Scaling and Cropping: An Application for Print-and-Scan Process," Multimedia and Security Workshop at ACM Multimedia, Orlando, FL, Oct 1999.

[21] F.A.P. Petitcolas, "Watermarking Schemes Evaluation,” IEEE Signal Processing, vol. 17, no. 5, pp. 58-64, September 2000.

http://www.cl.cam.ac.uk/ fapp2/publications/ieeespm00evaluation.doc

[22] F.A.P. Petitcolas, M. Steinebachb, F. Raynalc, J. Dittmannb, C. Fontained, N. Fatès, "A Public Automated WebBased Evaluation Service for Watermarking Schemes: StirMark Benchmark," in proc. Electronic Imaging, Security and Watermarking of Multimedia Contents, vol. 4314, San Jose, CA, January 2001.

http://www.cl.cam.ac.uk/ fapp2/publications/ei01automated.doc

[23] M. Schneider and S.-F. Chang, "A Robust Content Based Digital Signature for Image Authentication," IEEE Int. Conf. Image Processing, vol. 3, pp. 227-230, Sep. 1996.

[24] C.-S. Lu, C.-Y. Hsu, "Geometric Distortion-Resilient Image Hashing Scheme and Its Applications on Copy Detection and Authentication," Multimedia Systems, vol. 11, no. 2, pp. 159-173, 2005. 